\title{
Internal Expansive Reactions in Concrete Structures - Deterioration of the mechanical properties
}

\author{
João Custódio', António Bettencourt Ribeiro ${ }^{2}$ \\ ${ }^{1}$ LNEC - National Laboratory for Civil Engineering, Materials Department, Av. do Brasil, \\ 101, 1700-066 Lisbon, Portugal, jcustodio@lnec.pt \\ ${ }^{2}$ LNEC - National Laboratory for Civil Engineering, Materials Department, Av. do Brasil, \\ 101, 1700-066 Lisbon, Portugal
}

\begin{abstract}
A significant number of problems related to concrete deterioration have been detected in Portugal and worldwide as a result of the deleterious development of internal expansive reactions (IER). Their negative effect has important economic implications because they are the main cause of degradation of large concrete structures (e.g. dams and bridges). In addition, the work necessary to remediate the problem involves large areas of reconstruction and complex and expensive rehabilitation techniques and materials. Moreover, IER diminishes the affected structure service life, may involve the interruption of its function and, ultimately, can lead to its decommissioning and demolishing. The importance of these structures, the number of structures in which IER was already identified or is very likely to be diagnosed in a near future, the large number of structures that are under or planned for construction, which may also come to develop IER is why, today, they are still a major concern. Therefore, a study is being conducted at LNEC to diminish the negative impact of IER by increasing knowledge on how to reliably assess their extent and potential for future development in existing structures, so that the risks to structural integrity and need for mitigation/remediation actions can be properly assessed. To study the effect that internal expansion has in the deterioration of the concrete mechanical properties, the common practice is to perform tests on specimens exposed to an artificial environment that promotes IER. However, these tests take several months or even years to produce results. Thus, this communication presents the preliminary work carried out to devise a method capable of producing internal damage to concrete in a short period and in a way that the produced deterioration affects the concrete mechanical properties similarly to IER. From the several methods studied, one appears promising and it is detailed in the main body of the paper.
\end{abstract}

\section{Introduction}

In the last decade, a significant number of problems related to concrete deterioration were detected in large concrete structures in Portugal and throughout the world; the leading cause being internal expansive reactions (IER), more specifically, alkali-silica reaction (ASR) and internal sulphatic reaction (ISR).

ASR is a chemical reaction between the alkali hydroxides in the concrete pore solution and some siliceous minerals present within certain aggregates. The reaction results in the formation of a calcium-rich alkali-silica gel that is hydrophilic and expands in the presence of water causing the disruption of concrete $[1,2]$.

ISR is a chemical reaction between sulphate ions and calcium aluminates present in the hardened cement paste that results in the formation of ettringite. As the name suggests, the source of sulphate is in the concrete and can be the cement, the supplementary materials (e.g. fly ash), the aggregate, or the chemical admixtures. The formation of the so-called secondary or delayed ettringite has an expansive nature and can cause the disruption of concrete [2-4]. 